\title{
PAPEL DE CUIDADOR NO CONTEXTO DA HOSPITALIZAÇÃO DOMICILIÁRIA: DIAGNÓSTICO, IMPLEMENTAÇÃO E MONITORIZAÇÃO DE INTERVENÇ̃̃̃
}

\author{
Rui Dionísio \\ SIGMA - Phi Xi Chapter / ESSV / Politécnico de Viseu \\ Unidade de Saúde Pública \\ ACES Dão Lafões - Portugal \\ rmtdsps@hotmail.com \\ Cláudia Chaves \\ Doutoranda em Ciências de Enfermagem \\ Instituto de Ciências Biomédicas Abel Salazar \\ SIGMA - Phi Xi Chapter / CI\&DETS / \\ Politécnico de Viseu - Portugal \\ Paula Nelas \\ UICISA:E / CI\&DETS \\ Politécnico de Viseu - Portugal \\ Emília Coutinho \\ SIGMA - Phi Xi Chapter/ UICISA:E / CI\&DETS / \\ Politécnico de Viseu - Portugal \\ Carla Cruz \\ UICISA:E / CI\&DETS \\ Politécnico de Viseu - Portugal \\ Ana Andrade \\ UICISA:E / CI\&DETS \\ Politécnico de Viseu - Portugal
}

Fecha de Recepción: 7 Julio 2019

Fecha de Admisión: 25 Septiembre 2019

\section{RESUMO}

Hoje impõe-se um trabalho colaborativo e em rede com os profissionais das diversas unidades funcionais dos cuidados de saúde primários, dos cuidados hospitalares e da comunidade, pois só através de um sistema efetivo de referenciação e parceria, se conseguirá melhorar a acessibilidade, a qualidade, a continuidade e a eficiência dos cuidados de saúde, assim como a satisfação de profissionais e utilizadores.

Preconiza-se que os cuidados de saúde devam ser personalizados e de proximidade, o que implica, não só a deslocação de meios técnicos e humanos diferenciados de prestação de cuidados de saúde, mas também a sua prestação em tempo útil, aos utentes, família e cuidadores. Mas se 


\section{PAPEL DE CUIDADOR NO CONTEXTO DA HOSPITALIZAÇÃO DOMICILIÁRIA: DIAGNÓSTICO, IMPLEMENTAÇÃO E MONITORIZAÇÃ̃O DE INTERVENÇÃO}

uma maior proximidade permite uma melhor identificação de necessidades em saúde e 0 aumento de doenças crónicas requerem cuidados complexos e continuados no domicílio, então precisamos caraterizar que cuidadores temos e qual o seu papel enquanto cuidador no domicilio. Nesta perspetiva, o projeto proposto pretende realizar um diagnóstico de necessidades identificadas, pelos prestadores de cuidados informais, na região de Lafões, Portugal quando prestam cuidados a um doente e que espectativas têm dos profissionais dos cuidados de saúde primários de modo a ser possível a sua domiciliação, articulação e continuidade de cuidados. Para tal pretende-se constituir um estudo descritivo e de carácter exploratório utilizando como técnica de recolha de informação a entrevista, semiestruturada e tendo como metodologia base o questionamento circular. Posteriormente serão divulgados os resultados alcançados aos profissionais dos cuidados de saúde primários da região de Lafões e propor medidas e estratégias ao ministério da saúde de uma verdadeira articulação, continuidade e proximidade de cuidados tendo em conta as espectativas dos cuidadores assim como os recursos existentes na comunidade. Junto da população será efetuada sensibilização aos familiares e cuidadores das vantagens da permanência do doente em ambiente domiciliário e quais os recursos existentes.

Palavras-chave: hospitalização no domicílio; cuidador informal.

\section{ABSTRACT}

Career's role in the context of household hospitalization: diagnosis, implementation and monitoring of intervention. Today it is necessary to work collaboratively in a network with the professionals of the different functional units of primary health care, hospital care and community, because only through an effective system of referral and partnership will it be possible to improve the accessibility, quality, continuity and efficiency of health care, as well as the satisfaction of professionals and users. It is recommended that health care should be personalized and close, which implies not only the displacement of technical and human resources differentiated from the provision of health care, but also their provision in a timely manner to users, family and caregivers. But if proximity allows a better identification of health needs the increase of chronic diseases require complex and continuous care at home, and then we need to characterize the caregivers we have and what are their role as caregivers at home.

So, the proposed project intends to carry out a diagnosis of needs identified by informal caregivers in the region of Lafões, Portugal, when they provide care to a patient and what are the expectations of primary health care professionals and then be able to give them domiciliation, articulation and continuity of care. We intend to constitute a descriptive and exploratory study using semi-structured interviews as a technique for collecting information, based on circular questioning. Then we will be disseminate the results achieved to primary health care professionals in the Lafões region and propose measures and strategies to the Ministry of Health for a true articulation, continuity and proximity of care taking into account the expectations of the caregivers as well as the resources existing in the community. Subsequently, the family members and caregivers will be made aware of the advantages of the patient's stay in the home environment and the resources available to the population.

Keywords: hospitalization at home; informal caregiver.

\section{INTRODUÇÃO}

0 conceito de hospitalização domiciliária surgiu pela primeira vez em 1947, nos Estados Unidos da América com o projecto "Home Car", que tinha como principais objectivos, descongestionar os hospitais e criar um ambiente psicológico mais favorável para 0 doente (Delerue \& Correia, 2018). Na Europa, foi em França que se verificou em 1957 a primeira experiência. Em 1996, o Comité 
Regional da Europa da Organização Mundial da Saúde (OMS) promoveu o desenvolvimento do "Hospital para Cuidados de Saúde", também designado "Hospital em Casa", baseando-se no modelo americano (Cotta, Suárez-Varela, González, Cotta, Real, Ricós, 2001; Delerue \& Correia, 2018).

Vários estudos foram ocorrendo em que realçaram os benefícios dos cuidados de saúde em casa (Cotta et al., 2001; Lima, Spagnuolo, \& Patrício, 2013). Entre os benefícios enumerados há relevo também no factor económico, em que 0 valor do serviço resultou dos benefícios para os pacientes e cuidadores, e não da redução de custos aos hospitais especialmente no internamento de agudos (Brazil, Bolton, Ulrichsen \& Knott, 1998; Ince, Senturk, Singh, Yildiz, Danalio lu, Cinar, et al., 2014). As principais características de programas de rápido atendimento/acompanhamento domiciliário são enumeradas por Brazil et al. (1998) e ainda referem que estas vão além da tradicional resposta de cuidados de saúde na visitação domiciliária, tais como, a forte ligação entre 0 hospital e os serviços locais de assistência domiciliar, a avaliação e coordenação de serviços por um profissional de saúde do hospital, serviços domiciliares mais intensivos do que os normalmente oferecidos nos programas de atendimento domiciliar, tempo de resposta rápido desde a avaliação até ao cuidado no domicílio e serviço com limite de tempo (geralmente cinco dias).

Em Portugal, o Ministério da Saúde lançou em outubro de 2018 o projeto de hospitalização domiciliária com o objetivo de permitir aos doentes receber cuidados hospitalares no seu domicílio. De acordo com Despacho n.. 9323 (2018), que regulamenta a organização e funcionamento da Unidade de Hospitalização Domiciliária (UHD) em cada Hospital/Centro Hospitalar ou Unidade Local de Saúde, vários conceitos terão que ser tidos em conta, entre eles: Hospitalização domiciliária, Continuidade de cuidados, Familiar/es, Cuidador/es, a multidisciplinaridade e domicílio. 0 mesmo despacho determina a estratégia de implementação de Unidades de Hospitalização Domiciliária no Serviço Nacional de Saúde (SNS),garantindo o cumprimento de um "conjunto de critérios clínicos, sociais e geográficos que permitam a sua hospitalização no domicílio". Refere ainda que o "cuidar em casa deslocaliza a prestação dos cuidados das instituições para o domicílio, conduzindo assim a uma redefinição dos processos de cuidados e a uma estreita colaboração entre os vários intervenientes, desde os cuidados de saúde primários, aos hospitais e à Rede Nacional de Cuidados Continuados Integrados (RNCCl)".

Poderão ainda ser apontadas outras vantagens relacionadas com a humanização dos cuidados, a menor taxa de mortalidade, a redução das taxas de infecção hospitalar, assim como a diminuição do número de reinternamentos. Contudo, para que a hospitalização domiciliária ocorra terá que existir o consentimento do doente e da respetiva família e, se o doente não for autónomo, é preciso um cuidador exceto se 0 doente for autónomo nas atividades de vida diária (DGS, 2018). Apesar de estar previsto no âmbito da Rede de Cuidados Continuados Integrados, o reconhecimento e apoio aos Cuidadores Informais que apoiam as pessoas em situação de dependência nos seus domicílios, independentemente da idade, acreditamos que para o cuidador seria importante a existência de um maior e melhor apoio e para tal conhecer as suas necessidades e expectativas torna-se imperativo.

0 cuidador informal tem um papel complexo, na medida em que assume 0 desempenho da prestação de cuidados em três necessidades de cuidado ao idoso dependente: Apoio na informação e orientação de técnicas para facilitar 0 cuidado; Apoio emocional, com o objetivo fundamental de partilha e afeto trabalhando para a construção e manutenção da autoestima e por fim o apoio instrumental refere-se a todo tipo de cuidados que se devem prestar ao idoso dependente (Sequeira, 2010). 0 mesmo autor refere que o cuidador é uma pessoa que se caracteriza pelo envolvimento com 0 outro, vivendo numa contínua experiência de aprendizagem e descoberta das potencialidades mútuas em que 0 ambiente onde o idoso dependente está inserido, ou seja, as ajudas existentes, condições habitacionais e meios económicos são indicadores que influenciam o desempenho do cuidado. 


\section{PAPEL DE CUIDADOR NO CONTEXTO DA HOSPITALIZAÇÃO DOMICILIÁRIA: DIAGNÓSTICO, IMPLEMENTAÇÃO E MONITORIZAÇÃ̃O DE INTERVENÇÃO}

De modo a que o cuidador adquira conhecimentos, técnicas, estratégias e competências para garantir o seu equilíbrio e o da pessoa a ser cuidada terão que existir estratégias interventivas articuladas com os profissionais de saúde, serviço social entre outros, desenvolvendo um trabalho em parceria (Sequeira, 2010; Mata \& Rodríguez, 2012; Schaepe \& Ewers, 2018). É importante a avaliação das dificuldades do cuidador, pois esta possibilita a adequação das respostas e apoios sociais (Sequeira, 2010). Nesta perspetiva, o cuidador principal e informal será a pessoa (familiar ou não) que coabita ou não com o doente, e que Ihe oferece a maior parte dos cuidados (físicos e emocionais) e apoio diário, não sendo remunerada por esta actividade (Escudero Rodriguéz, Díaz Álvarez \& Pascual Cortés, 2001).

Sabendo que a necessidade está relacionada a uma noção de valor, estando dependente do significado que cada pessoa atribui, do contexto em que está inserida, dos valores e das crenças, diversos estudos sugerem que as necessidades da família diferem, de um modo significativo, das necessidades do doente (Messeger, 1998; Farber et al., 2003; Hudson, Aranda \& Kristjanson, 2004). Para melhor compreendermos as famílias e integrar esta informação nos cuidados que se prestam aos indivíduos, surgiu a Teoria Geral dos Sistemas, em que a família, que tem o membro doente é vista como um sistema aberto em interacção com o sistema de prestação de cuidados de saúde, reagindo e adaptando-se às informações e intervenções dos prestadores de cuidados. Sampaio e Gameiro (1988), adianta-nos que a família é o sistema utilizado mais frequentemente para a resolução dos problemas. Deve ser entendida de uma forma dinâmica, sofrendo modificações ao longo do tempo de modo que consiga atingir um equilíbrio entre duas tendências homeostasia e a transformação. Pela capacidade homeostática a família mantém a sua unidade, pela transformação, a família adapta-se sucessivamente às necessidades do seu percurso longitudinal.

Na avaliação de uma família é útil considerar ainda a dimensão da coesão e da comunicação. Olson et al (1983), defende que a coesão está relacionada com o grau de investimento e suporte que os membros da família têm uns relativamente aos outros, medindo o elo emocional que os une. McCain (1998), refere que hoje em dia, as pessoas vivem durante mais tempo depois de ter sido feito o diagnóstico, e os filhos adultos dispensam mais cuidados aos idosos, cuidados assegurados com frequência pelas filhas e noras. Menciona ainda que nos Estados Unidos e no Canadá, são os membros da família que prestam a maioria dos cuidados domiciliários aos idosos dependentes. No decorrer da hospitalização, 0 alvo dos cuidados deve ser tanto 0 doente como aquele que lhos assegura. Uma vez que o mesmo com incapacidade depende daqueles que cuidam dele, é indispensável dar informações aos que o acompanham, de forma a assegurar continuidade na assistência após a alta.

Em suma a doença de modo geral é tida como uma crise no seio da família podendo conduzir alterações interiores (de status familiar, da definição de regras, da quantidade e qualidade da actividade sexual, entre outras), ou exteriores (contactos sociais ou profissionais). Por norma, quanto melhor for 0 nível de organização e integração prévia à situação de doença, melhor será a forma da família em lidar e adaptar-se aos novos problemas consequentes (Relvas, 1996). A crescente incapacidade para "cuidar dos seus" deve-se a questões de ordem interna e a alterações dos padrões morfológicos da família assim como a alterações sociais das últimas décadas, por exemplo, a relação com o mundo do trabalho e as estâncias responsáveis da prestação de cuidados (Portugal, 2000). Alarcão (2000), insiste na necessidade em considerar as redes sociais como instrumento de trabalho como setting terapêutico de forma a não abandonar o conceito de sistema familiar.

Como facilmente se depreende a família nuclear e até a alargada, constitui não só uma parte importante da rede social do indivíduo como representa a sua primeira rede. Nela o idoso dependente está contido e por tal facto torna-se imperativo abordar o que se entende por rede social de apoio e qual 0 seu papel no bem-estar dos mesmos quando se aborda a hospitalização no domicilio. 


\section{MATERIAL E MÉTODOS}

Objetivos: Este projecto apresenta como objectivo geral, identificar as necessidades dos cuidadores informais e que expectativas têm dos profissionais dos cuidados de saúde primários. E para a consecução deste, há que atingir de forma escalar os seguintes objectivos específicos: avaliar as dificuldades sentidas pelo cuidador informal; avaliar e compreender o impacte da prestação de cuidados na família no cuidador informal; compreender a experiencia dos cuidadores informais durante o processo de prestação de cuidados; compreender o processo de continuidade e articulação dos cuidados de saúde entre os profissionais e o cuidador na hospitalização domiciliária.

Desenho do estudo: abordagem de metodologia mista, observacional, descritivo e transversal.

Local e Horizonte temporal: Recolha da amostra realizada durante $02^{\circ}$ semestre de 2019 e 1은 semestre de 2020 nos centros de saúde da região de Lafões, Portugal.

População: Cuidadores informais que prestem cuidados há pelo menos 6 meses ou que estejam no programa de Hospitalização domiciliária na região de Lafões.

Tipo de amostragem e dimensão da amostra: A amostragem é não probabilística e de conveniência, uma vez que não se pretendem generalizar os seus resultados, tratando-se de um estudo exploratório no qual participarão os cuidadores que acederem em fazê-lo e que forem referenciados pelos médicos ou enfermeiros dos centros de saúde da região de Lafões, prevendo-se a participação de 80 cuidadores.

Método de recolha de dados: Pretende-se contactar todas as equipas e profissionais pertencentes aos centros de saúde da região de Lafões. Para tal e tendo em conta a necessidade de uma reunião com os coordenadores das Unidades Funcionais (Unidade de Saúde Familiar, Unidade de Cuidados Personalizados, e Unidade de cuidados na Comunidade) pretende-se contactar o Director do Agrupamento de Centros de Saúde do Dão Lafões no sentido de apresentar o trabalho a realizar e solicitar uma reunião com os médicos e enfermeiros dos respetivos centros de saúde. Na reunião com os profissionais pretende-se apresentar os objetivos do trabalho. Em relação à recolha da amostra, será pedido aos enfermeiros que consultem os seus registos de forma a listar os cuidadores informais de doentes há mais de 6 meses assim como os doentes em contexto de Hospitalização domiciliária. Posteriormente, será solicitado aos cuidadores informais o preenchimento de um questionário. Neste processo será assegurado 0 anonimato quer dos cuidadores, quer dos doentes.

Nesta pesquisa, e dado tratar-se de um estudo exploratório descritivo de de tipologia mista, tivemos em conta: variáveis de atributo e dados obtidos em função das questões de investigação e dos objectivos de estudo. As variáveis de atributo que consideramos mais relevantes para a caracterização sociodemográfica da amostra são: sexo, idade, estado civil, situação profissional, habilitações literárias, local de residência, nível económico do agregado familiar, data de ocorrência da doença e grau de parentesco relativamente ao doente. A informação que se pretende obter com este trabalho está relacionada com: as alterações ocorridas na família quando surgiu a doença, a relação familiar e redes de suporte, as necessidades identificadas pelo cuidador informal na prestação de cuidados e na articulação e continuidade de cuidados.

\section{RESULTADOS ESPERADOS}

Acreditamos que os sistemas de saúde estão confrontados com desafios em que a integração de cuidados é uma delas. Deve-se proporcionar um maior investimento na coordenação e articulação no sistema de saúde, facultando melhor integração e coordenação entre os diversos níveis de cuidados e efetuar novas abordagens organizacionais adequando a capacidade de resposta às populações mais carenciadas. 


\section{PAPEL DE CUIDADOR NO CONTEXTO DA HOSPITALIZAÇÃO DOMICILIÁRIA: DIAGNÓSTICO, IMPLEMENTAÇÃO E MONITORIZAÇÃ̃O DE INTERVENÇÃO}

Teremos que apostar numa mudança de paradigma incentivando a transferência de recursos hospitalares para a comunidade. 0 ambulatório produz melhorias ao nível da qualidade assistencial e a um aumento da eficiência técnica e económica. Beneficiaríamos se conseguíssemos rentabilizar os recursos locais, estabelecendo parcerias e respostas adequadas à comunidade assim como estabelecer acordos de cooperação entre os cuidados hospitalares e primários de modo rentabilizar os recursos existentes. Com a iniciativa da hospitalização domiciliária reforça-se a necessidade da construção da identidade do estatuto do cuidador informal. Nesta perspetiva acreditamos que com este trabalho, iremos aprofundar conhecimento sobre as necessidades e expectativas do cuidador informal da região de Lafões mas também constatar todo o efeito na área da articulação e da continuidade de cuidados os diversos níveis de cuidados e entre as várias unidades dos cuidados de saúde primários, designadamente através das unidades de saúde familiar (USF), das unidades de cuidados de saúde personalizados (UCSP), das unidades de cuidados na comunidade (UCC), da hospitalização domiciliária, das equipas de cuidados continuados integrados (ECCI) da RNCCI, das respostas de serviço social e de outros agentes da comunidade.

\section{CONSIDERAÇÕES FINAIS}

Recomendamos que o cuidador informal tem que ser reconhecido como uma mais-valia e um parceiro na prestação de cuidados. Para tal, capacitá-lo através da formação e informação e envolve-lo através da atenção, participação e gestão de expectativas e necessidades constituirão desafios que todos os prestadores de cuidador formais terão que incluir nas suas atividades. Este trabalho pretende, assim, contribuir para melhor conhecer o cuidador informal e desta forma ser reconhecido como um parceiro estratégico na arte do cuidar.

Esperamos, poder compreender que necessidades e espectativas o cuidador tem nos profissionais dos cuidados de saúde primários e quais, as dificuldades que surgiram na articulação e continuidade de cuidados. Espera-se, assim, que a análise dos dados recolhidos possa contribuir para que no futuro a prestação de cuidados se centre também nas necessidades identificadas pelo cuidador informal, pois desta forma estariam os profissionais de saúde a responder ao doente e a quem cuida no seu domicílio e ao mesmo tempo identificar processos de melhoria ao nível da continuidade e articulação de cuidados entre os diversos níveis.

Acreditamos que 0 impacte deste trabalho possibilitará um maior conhecimento sobre as características dos cuidadores informais que prestam cuidados no domicílio e desta forma chamar a atenção para a necessidade de se implementar definitivamente 0 estatuto do cuidador informal e alertar para uma política de formação permanente em contexto domiciliário por parte de equipas dos cuidados de saúde primários, de modo a possibilitar um adequado apoio continuo aos cuidadores informais. Definitivamente deve apostar-se numa verdadeira política voltada para a qualidade que só se conseguirá dando maior enfase na prevenção e na aproximação dos cuidados de saúde aos cidadãos através do reforço dos cuidados de saúde primários.

\section{REFERÊNCIAS BIBLIOGRÁFICAS}

Alarcão, M. (2000). (Des)equilíbrios familiares: Uma visão sistémica. Coimbra: Quarteto.

Brazil, K., Bolton, C., Ulrichsen, \& D. Knott, C. (1998). Substituting home care for hospitalization: The role of a quick response service for the elderly. Journal of Community Health, 23(1), 2943. Acedido em https://DOI.ORG/10.1023/A:1018770820728

Cotta, R., Suárez-Varela, M., González, A., Cotta, J., Real, E., \& Ricós, J. (2001). La hospitalización domiciliaria: Antecedentes, situación actual y perspectivas. Revista Panamericana de Salud Pública, 10(1), 45-55. Acedido em https://scielosp.org/pdf/rpsp/2001.v10n1/45-55/es

Delerue, F., \& Correia, J. (2018). Hospitalização domiciliária mais um desafio para a medicina inter- 
na. Medicina Interna, 25(1), 15-17. Acedido em http://dx.doi.org/10.24950/rspmi/0p/1/2018

Despacho n.- 9323-A/2018. (2018, outubro 3). Modelo de deliberação de criação e de regulamento da Unidade de Hospitalização Domiciliária. Diário da República, 2(191), pp. 26900-(2) a 26900(5). Acedido em https://dre.pt/home/-/dre/116587923/details/maximized

Escudero Rodríguez, B., Díaz Álvarez, E., \& Pascual Cortés, 0. (2001). Cuidadores informales: Necesidades y ayudas. Revista Rol de Enfermería, 24(3), 183-189.

Farber, S. J., Egnew, T. R., Herman-Bertsch, J. L., Taylor, T. R., \& Guldin, G. E. (2003). Issues in endof-life care: Patient, caregiver, and clinician perceptions. Journal of Palliative Medicine, 6(1), 1931. Acedido em https://doi.org/10.1089/10966210360510082

Ince, A. T., Senturk, H., Singh, V. K., Yildiz, K., Danalio lu, A., Cinar, A., ... Gürakarb, A. (2014). A randomized controlled trial of home monitoring versus hospitalization for mild non-alcoholic acute interstitial pancreatitis: a pilot study. Pancreatology, 14(3), 174-178. Doi: 10.1016/j.pan.2014.02.007.

Lima, A. A., Spagnuolo, R. S., \& Patrício, K. P. (2013). Revendo estudos sobre a assistência domiciliar ao idoso. Psicologia em Estudo, 18(2), 343-351. https://dx.doi.org/10.1590/S141373722013000200015

Mata, M. A. P., \& Rodríguez, M. T. V. (2012). El cuidado no remunerado de mayores dependientes en el el noreste de Portugal. Prisma Social: Revista de Ciencias Sociales, 8, 333-357. Acedido em http://www.redalyc.org/articulo.oa?id=353744580011

McCain, G. C. (1998). A família. In V. B. Bolander (ed.lit.), Enfermagem fundamental: abordagem psicofisiológica (Cap. 18, pp. 397-408). Lisboa: Lusodidacta.

Messeger, L. (1998). Preocupaciones y necesidades de la familia del enfermo en fase terminal. In Enfermería en cuidados paliativos (pp. 237-243). Madrid: Panamericana.

Olson, D. H., McCubbin, H. I., Barnes, H., Larsen, A., Muxen, M., \& Wilson, M. (1983). Families Inventoris. St. Paul, Minnesota: Family Social Science, University of Minnesota.

Portugal, Ministério da Saúde, Direção Geral da Saúde. (2018). Norma no 020/2018: Hospitalização domiciliária em idade adulta. Lisboa: DGS. Acedido em https://www.dgs.pt/directrizes-dadgs/normas-e-circulares-normativas/norma-n-0202018-de-20122018.aspx

Portugal, S. (2000). Retórica e acção governativa na área das políticas de família desde 1974. Revista Critica de Ciências Sociais, 56, 81-98. Acedido em https://www.ces.uc.pt/publicacoes/rccs/artigos/56/Silvia\%20Portugal\%20\%20Politicas\%20de\%20familia.pdf

Relvas, A. P. (1996). A co-construção da hipótese sistémica em terapia familiar. Análise Psicológica, 14(4.), 563-579. Acedido em http://repositorio.ispa.pt/handle/10400.12/3180

Sampaio, D., \& Gameiro, J. (1988). Avaliação sistémica da família: Texto de apoio à acção de formação «Família: saúde e doença». Lisboa: ICGS.

Schaepe, C., \& Ewers, M. (2018). "I see myself as part of the team": Family caregivers' contribution to safety in advanced home care. BMC Nursing,17(40). Doi: 10.1186/s12912-018-0308-9.

Sequeira, C. (2010). Cuidar de idosos com dependência física e mental. Lisboa: Lidel 
